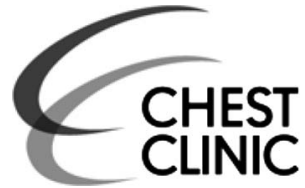

${ }^{1}$ National Pulmonary Hypertension Service, Royal Brompton Hospital, London, UK

${ }^{2}$ Department of Medical Oncology, Charing Cross Hospital, London, UK

Correspondence to Dr Laura Price, National Pulmonary Hypertension Service, Royal Brompton Hospital, Sydney Street, London SW3 6NP, UK; laura.price@rbht.nhs.uk

Received 4 April 2016 Revised 4 June 2016 Accepted 6 June 2016 Published Online First 1 July 2016
CrossMark

To cite: Tyebally $S$,

Ghorani E, Kaur B, et al.

Thorax 2016;71:871-872.

\title{
A case of progressive breathlessness post partum
}

\author{
Sara Tyebally, ${ }^{1}$ Ehsan Ghorani, ${ }^{2}$ Baljeet Kaur, ${ }^{2}$ Ann Sturdy, ${ }_{1}^{1}$ Arif Khokhar, ${ }^{1}$ \\ Rosemary Fisher, ${ }^{2}$ Michael Seckl, ${ }^{2}$ Laura Price $^{1}$
}

\section{CLINICAL PRESENTATION}

A 35-year-old woman presented with rapidly progressive dyspnoea (WHO functional class III) two months after normal vaginal delivery at term, with no other symptoms. On examination, she was normotensive, with a raised jugular venous pressure, right ventricular (RV) heave, a prominent pulmonary second heart sound and mild pitting leg oedema. Oxygen saturations were $90 \%$ on air with a clear chest on auscultation. There was a single enlarged supraclavicular lymph node.

ECG showed sinus tachycardia with right axis deviation. Renal and liver function were normal but brain natriuretic peptide and $\mathrm{D}$-dimer were raised. Chest radiograph showed clear lungs and enlarged proximal pulmonary arteries, with no filling defects or parenchymal disease evident on CT pulmonary angiogram (CTPA). Transthoracic echocardiogram revealed signs of severe pulmonary hypertension $(\mathrm{PH})$ with severely impaired RV function. Right heart catheterisation confirmed precapillary $\mathrm{PH}$ with a mean pulmonary artery pressure of $39 \mathrm{~mm} \mathrm{Hg}$, pulmonary wedge pressure of $13 \mathrm{~mm} \mathrm{Hg}$, cardiac output of $2.1 \mathrm{~L} / \mathrm{min}$ and pulmonary vascular resistance of 12.4 Wood units, without reversibility to inhaled nitric oxide.

At this point, assessment was thought to reflect 'moderate risk' chronic thromboembolic PH (CTEPH). She was anticoagulated, and commenced on upfront combination $\mathrm{PH}$ therapy ${ }^{1}$ with a phosphodiesterase inhibitor (tadalafil) and endothelin receptor antagonist (macitentan). She developed increasing frontal headache, thought to be a consequence of $\mathrm{PH}$ therapy. Her headache persisted, despite weaning $\mathrm{PH}$ therapies, with associated vomiting but no localising neurological signs, and a CT of the head was performed.

\section{QUESTION}

What is the underlying diagnosis, as suggested by the images below (figure $1 \mathrm{~A}, \mathrm{~B}$ )?
A

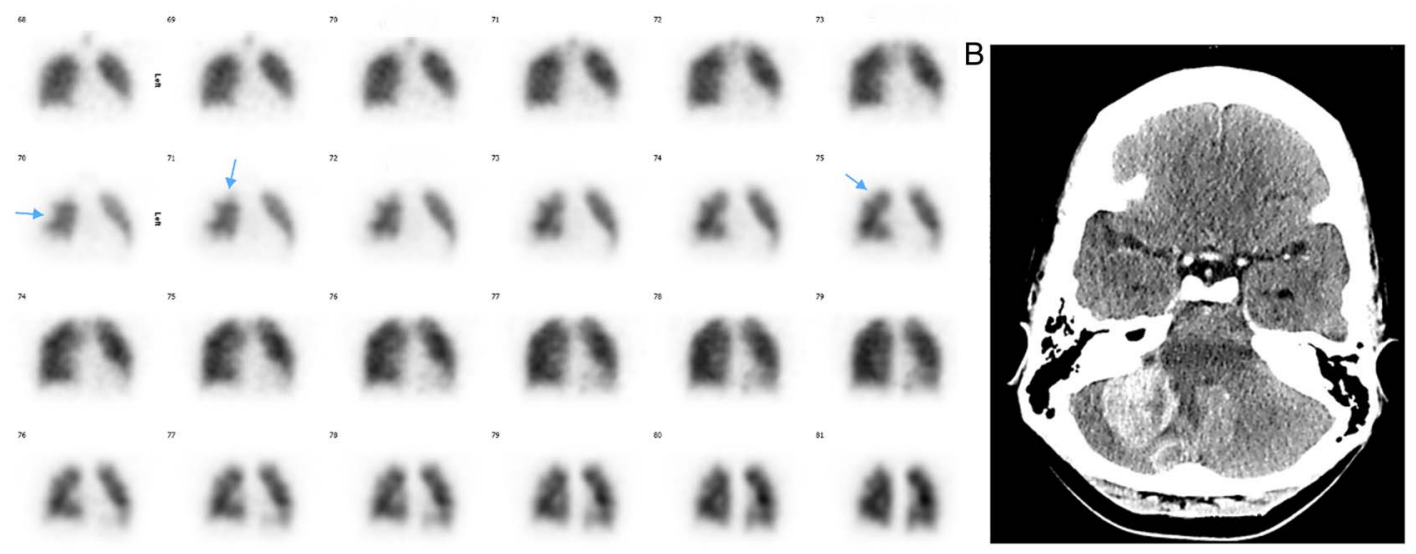

Figure 1 (A) Ventilation perfusion (VQ) scan at presentation showing multiple small peripheral subsegmental defects in the apical, anterior and posterobasal segments of the right lung. Perfusion in much of the left lung is well maintained. The ventilation images show relatively homogeneous distribution of tracer in both lungs. (B) CT of the head with intravenous contrast showing several high-density lesions in the posterior fossa, which enhance strongly post administration of intravenous contrast, with surrounding oedema. 


\section{ANSWER}

CT of the head suggested brain metastases, and the diagnosis of choriocarcinoma was later confirmed on supraclavicular lymph node core biopsy. PH was initially thought to reflect distal CTEPH until the diagnosis of malignancy evolved, with likely pulmonary tumour embolism (PTE) relating to choriocarcinoma. $^{2}{ }^{3}$ The finding of a raised D-dimer (outside the immediate post-partum period) with severe $\mathrm{PH}$, a normal CTPA and distal perfusion defects on ventilation perfusion (VQ) scan is suggestive of tumour microemboli ${ }^{4}$ and should raise the suspicion of malignancy.

Malignancy-induced PH due to tumour microemboli comprises a likely spectrum of PTE and pulmonary tumour thrombotic microangiopathy (PTTM), and the distinction between PTE and PTTM is only possible following histological examination. In PTE, coagulated tumour cells obstruct pulmonary arteries. In PTTM, additional fibrointimal proliferation occludes pulmonary arteries, lymphatics and veins. PTTM is usually associated with carcinomas, often gastrointestinal adenocarcinomas, and is uniformly fatal. ${ }^{4}$ PTE can resolve depending on the chemosensitivity of the underlying tumour. ${ }^{2}$

Choriocarcinomas may be gestational or non-gestational in origin and are potentially very chemosensitive. ${ }^{6}$ Prompt diagnosis is therefore crucial to enable access to chemotherapy. Our patient had an excellent initial response to emergency cisplatinbased chemotherapy, with improvement in tumour markers and $\mathrm{PH}$, although stable perfusion defects on repeat VQ. Two months following diagnosis, she developed resistance to secondline chemotherapy, suggesting that this was a non-gestational tumour and the PH suddenly worsened. She died of a PH crisis 3 months after the initial diagnosis. Genetic analysis confirmed a non-gestational tumour. ${ }^{6}$

Clues to the diagnosis of PTE/PTTM in this case included the presence of a raised serum D-dimer without proximal filling defects on CTPA but distal defects on VQ, with rapid onset severe $\mathrm{PH}$, and the history of recent pregnancy. The use of tumour markers in a patient presenting with $\mathrm{PH}$ is not routine, but the finding of grossly raised serum and urine $\beta$ human chorionic gonadotropin may have led to an earlier diagnosis in this case, as previously reported. ${ }^{2}$ Choriocarcinoma is also reported following spontaneous abortions and especially in women with previous hydatiform moles, ${ }^{6}$ which may be relevant if risk stratifying other young woman in this situation. This case emphasises the importance of consideration of malignant aetiology in a patient presenting with $\mathrm{PH}$, and the potential impact of prompt diagnosis upon oncological outcomes.

Acknowledgements Dr Rafael Alonso-Gonzalez, Dr Konstantinos Dimopoulos, Dr John Wort and Carl Harries, National Pulmonary Hypertension Service, Royal Brompton Hospital Dr Luke Howard, Pulmonary Hypertension Physician, Hammersmith Hospital Dr Kshama Wechalekar, nuclear medicine physician, Royal Brompton Hospital Ms Dee Short, Oncology service manager, Charing Cross Hospital

Competing interests None declared.

Patient consent Obtained.

Provenance and peer review Not commissioned; externally peer reviewed.

\section{REFERENCES}

1 Galiè N, Humbert M, Vachiery JL, et al. 2015 ESC/ERS Guidelines for the diagnosis and treatment of pulmonary hypertension: The Joint Task Force for the Diagnosis and Treatment of Pulmonary Hypertension of the European Society of Cardiology (ESC) and the European Respiratory Society (ERS): Endorsed by: Association for European Paediatric and Congenital Cardiology (AEPC), International Society for Heart and Lung Transplantation (ISHLT). Eur Heart J 2016;37:67-119.

2 Seckl MJ, Rustin GJ, Newlands ES, et al. Pulmonary embolism, pulmonary hypertension, and choriocarcinoma. Lancet 1991;338:1313-15.

3 Hibbert M, Braude $\mathrm{S}$. Tumour microembolism presenting as "primary pulmonary hypertension". Thorax 1997;52:1016-17.

4 Pinckard JK, Wick MR. Tumor-related thrombotic pulmonary microangiopathy: review of pathologic findings and pathophysiologic mechanisms. Ann Diagn Pathol 2000;4:154-7.

5 Chinen K, Tokuda Y, Fujiwara M, et al. Pulmonary tumor thrombotic microangiopathy in patients with gastric carcinoma: an analysis of 6 autopsy cases and review of the literature. Pathol Res Pract 2010;206:682-9.

6 Seckl MJ, Sebire NJ, Berkowitz RS. Gestational trophoblastic disease. Lancet 2010;376:717-29. 